\title{
The unsuspected capacity of melanin to transform light energy into chemical energy and the surprising anoxia tolerance of chrysemys picta
}

\begin{abstract}
Oxygen is considered critical to nearly all life on earth, as the end electron acceptor that makes, theoretically, mitochondrial oxidative phosphorylation possible, and thereby energy production. Anaerobic energy sources can only temporarily supply ATP and maintain cellular function before substrate depletion, energy shortfall, or end-product poisoning that threaten survival. In most vertebrates, the limits of anoxia tolerance are short, of the order of minutes, because of the urgent dependence (theoretically) of the heart and central nervous system on a continuous supply of $\mathrm{O}_{2}$.

Because greater than $50 \%$ of the energy consuming processes of the normoxic cell, especially neuron can be accounted for by ion pumping, a decrease in membrane ion leakage can contribute in a major way to energy savings. Theoretically, the power to the inside of the cell depends on ATP, but in the light of our discovery of the intrinsic property of melanin transform light energy into chemical energy by means of the water molecule dissociation, we think that the levels of ATP, in turn; depend almost completely from the energy that emanates from the melanin.

The purpose of discerning the ability of the painted turtle, of tolerating extended periods of anoxia, even months, will improve our understanding of the pathophysiology and progress in therapeutic strategies in diseases that can be classified as events hypoxic severely affecting human beings, such as cerebral vascular events.
\end{abstract}

Volume 2 Issue 3 - 2015

\section{Arturo Solis Herrera}

Centro de Estudios de la Fotosintesis Humana, México

Correspondence: Arturo Solis Herrera, Centro de Estudios de la Fotosintesis Humana, México, Tel 0I4499|60048, 014499|50042, Email comagua2000@yahoo.com

Received: July 19,2015 | Published: August 24, 2015

Keywords: melanin, photosynthesis, anoxia, hypoxia, chrysemys picta

Abbreviations: RER, rough endoplasmic reticulum; SER, smooth endoplasmic reticulum

\section{Introduction}

Molecular oxygen $\left(\mathrm{O}_{2}\right)$ is essential for vertebrate life. Strikingly, certain species are able to survive for periods lasting several months in anoxic conditions and are able to recover full function at the end of this time when $\mathrm{O}_{2}$ is restored. The freshwater turtle Chrysemys picta is a well-studied example. It spends long periods during the winter in ice-covered ponds without access to the surface, often in water or mud with little or no $\mathrm{O}_{2}$.

The painted turtle, under experimental conditions, can survive continuous submergence in nitrogen-equilibrated water at $3^{\circ} \mathrm{C}$ for more than 4months. ${ }^{1}$ This extraordinary resistance to the hypoxia has attracted much attention, so it has been the subject of numerous studies that have tried to explain it.

It is thought that a low metabolic rate is a key piece to tolerate a so prolonged anoxia. Being an ectothermic reptilian, its metabolism is only $10-20 \%$ in comparison to a mammal of similar temperature and size. At low temperatures, its metabolism decreases at a rate of 2 to 3 folds for each $10^{\circ} \mathrm{C}$ temperature. At a temperature of $3^{\circ} \mathrm{C}$, aerobic metabolism drops to around $0.1 \%$ in relation to a mammal. In anoxic state metabolism is 10000times less than that of a mammal of similar size. Numbers show huge differences between reptiles and mammals, and however, at the biochemical level, the differences are subtle, they do not explain such discrepancy. Moreover, reptiles with shells, are the ones who most resist anoxia, non-shelled reptiles, not.

The low rate of metabolism slows the build-up of acid endproducts. However, this metabolism so attenuated, theoretically should be able to supply the necessary energy for the turtle during anoxia of hibernation. In this state, the animals respond to stimuli and move very slowly periodically.

Experimental evidence from anoxic turtles indicates a coordinated downregulation in the rates of both ATP utilization and ATP production. The cellular ATP levels remain stable during long periods of anoxia; however, the involved mechanisms are still unknown. Studies in anoxic turtle have focused mainly on the brain because of the critical importance of maintaining this organ's function. It is not understood why the brain of the turtle does not show the hypoxic damage observed in the brain of mammals. The explanation about why in turtle does not happen the collapse of the Ionic gradients, as in mammals, is poorly understood and there are only complicated theories trying to explain it. Decoding the way as it protects the nervous tissue of the turtle painted during anoxia would make it possible to design therapeutic strategies applicable to humans, because the aftermath of hypoxic or anoxic minutes events, severe damage the central nervous system in mammals and humans, leaving permanent sequelae.

It is paradoxical that some proteins increase its synthesis in the heart and liver of turtle during anoxia, while decreasing production and use of the ATP that's occurring during anoxia. Furthermore, turtle, 
theoretically, during hibernation, changes its metabolism to anaerobic, i.e. the production of ATP via glycolysis, which is a very inefficient metabolic pathway, as it requires large amounts of substrate in the form of glucose or glycogen. The above is not possible explain it in a consistent manner unless you consider the possibility of a different, unknown power source, either unsuspected.

A challenge for an anoxia-tolerant animal is the accumulation of high concentrations of lactate and the associated burden of protons. Some species of fish produces ethanol as the principal anaerobic end-product, however, all vertebrate tetra pod produce lactate as the glycolytic end-product. When ATP synthesis equals ATP hydrolysis, ATP hydrolysis produces protons in a 1:1 with lactate production.

After 3-5months of experimental submergence at $3^{\circ} \mathrm{C}$, circulating lactate levels in turtle can reach 150-200 mmol 1-1. The acid load of this amount of lactate greatly exceeds normal body fluid buffering capacity thereby requires substantial supplemental buffering to prevent fatal acidosis. The observation that the shell, the turtle's most distinctive anatomical feature, and skeleton of the turtle serve as the sources of this additional buffering as well as a sink for lactic acid, are relatively recent explanations of the extraordinary tolerance to anoxia turtle, however, we must take into account the melanin present in the shell.

Something they have in common the researchers who have studied the surprising strength of the tortoise to anoxia, is that they have not taken into account the pigment of the carapace, melanin; reflecting the widespread misconception that is not more than a simple built-in sunscreen that protects us from dangerous UV rays.

The surprising ability of the painted turtle tolerate anoxia for prolonged periods has been studied from various points of view, but a constant in these works is the lack of attention to the molecule of melanin in skin and shell. It does not seem to draw the attention of the researchers the insistence of nature in place on the carapace and skin of virtually all turtles.

\section{Background}

In mammalian brain, excitotoxic cell death occurs within minutes of exposure to anoxic conditions. Excitatory neurotransmitters, primarily glutamate and dopamine, are rapidly released, because the highly complex order of cell, e.g. neuron; requires certain chemical energy levels available in order to maintain it, and when chemical energy levels are downed, then there is a markedly disorganization or even disruption in use of the scarce available chemical energy; being calcium influx alterations a clear example of generalized imbalance. The elevation in extracellular transmitter levels comes about as the result of a decrease in reuptake as well as an increase in both vesicular and non-vesicular release. The decrease in reuptake can be explained as reuptake needs chemical energy available, and the vesicular and non-vesicular release also requires energy. Neurons are generally viewed as among the most anoxia sensitive of all cells, though recent studies have shown a wide variation in the capacity of neurons to tolerate hypoxia, reflective of function and the degree of hypoxia normally encountered.

Freshwater turtles of the genera Trachemys and Chrysemys are true facultative anaerobes, able to survive from up to $48 \mathrm{hrs}$ at room temperature to months (during winter hibernation) in the total absence of oxygen. ${ }^{2}$ By preventing an energy deficit, the turtle brain avoids the catastrophic drop in ATP levels which in mammalian neurons results in the breakdown of cellular ion homeostasis, release of excitatory neurotransmitters, and excitotoxic cellular death. ${ }^{3}$ Such extended anoxic survival time is not a matter of ectothermy.

The turtle must maintain, over days to weeks, the integrity of the brain in a state of deeply depressed metabolism at an order of magnitude lower than in normoxic. This cannot be a matter of simply "shutting off" the brain, however, as the function and integrity of the neural network must be maintained and in a state of readiness for recovery.

Trachemys scripta has been the subject of extensive research into the adaptations that permit neuronal survival without oxygen, but none of the published studies seems to take into account the ever presence of melanin that the turtle has mainly on the skin and in the shell, at least macroscopically. Therefore, their conclusions are confuses, intricate and ultimately do not resolve the question. Thus at no time do turtle neurons experience an energy deficit that would otherwise constitute a trigger for catastrophic cell death, but this can be explained as consistent, if we add to the equation the unsuspected intrinsic property of melanin transform light energy into chemical energy by means of the dissociation of the molecule of water, such as chlorophyll in plants.

\section{Light energy transformation into chemical energy}

The very first step in plants can be represented as follows:

$$
\mathrm{H}_{2} \mathrm{O} \text { (liquid) } \rightarrow 2 \mathrm{H}_{2} \text { (gas) }+\mathrm{O}_{2} \text { (gas) }
$$

Traditionally gives much value to molecular oxygen is generated as a result of the dissociation of the water molecule, but in our opinion the value product is molecular hydrogen, since it is the carrier of energy par excellence in the entire universe, besides a powerful antioxidant effect that is very useful in biochemical terms, ${ }^{4}$ and on the other hand, oxygen is toxic to any concentration, proof of this is that the plants expel it into the atmosphere.

To date, chlorophyll was considered the only one biological molecule capable of carrying out such a transformation, but now that melanin makes its appearance as bioenergetic molecule of first order, the old paradigm of chlorophyll is broken into a thousand pieces.

Melanin, the mammal's chlorophyll: Melanin, luminous energy becomes chemical energy, ${ }^{5}$ as in chlorophyll happens, but is thousands of times more efficient given that melanin absorbs all of the electromagnetic spectrum, this is: from radio waves to gamma rays, and chlorophyll just absorbs the extreme of visible light, blue and red. In addition to that, unlike chlorophyll, in which the dissociation of water is irreversible, in the melanin the dissociation of the water molecule is reversible, i.e.: melanin transforms the liquid water in gas and vice versa, which can be represented in the following way:

$$
\mathrm{H}_{2} \mathrm{O} \text { (liquid) } \rightarrow 2 \mathrm{H}_{2} \text { (gas) }+\mathrm{O}_{2} \text { (gas) } \rightarrow \mathrm{H}_{2} \mathrm{O} \text { (liquid) }+4 \mathrm{e}-
$$

\section{Either}

$$
\mathrm{H}_{2} \mathrm{O} \text { (liquid) } \leftrightarrow 2 \mathrm{H}_{2} \text { (gas) }+\mathrm{O}_{2} \text { (gas) }+4 \mathrm{e}-
$$

For every two molecules of water that is re-formed, 4 high energy electrons are generated by what we obtain energy during dissociation and also the re-formation of the water molecule. Part of the energy that is released as a result of the dissociation of the water molecule, is captured by the hydrogen molecule, which carries it (Figure 1). Since the hydrogen molecule is not combined with the water, it moves through it, and in the case of the cell, scrolls through the cytoplasm, allowing it to reach up to the last corner of the cell (Figure $2 \& 3$ ). 


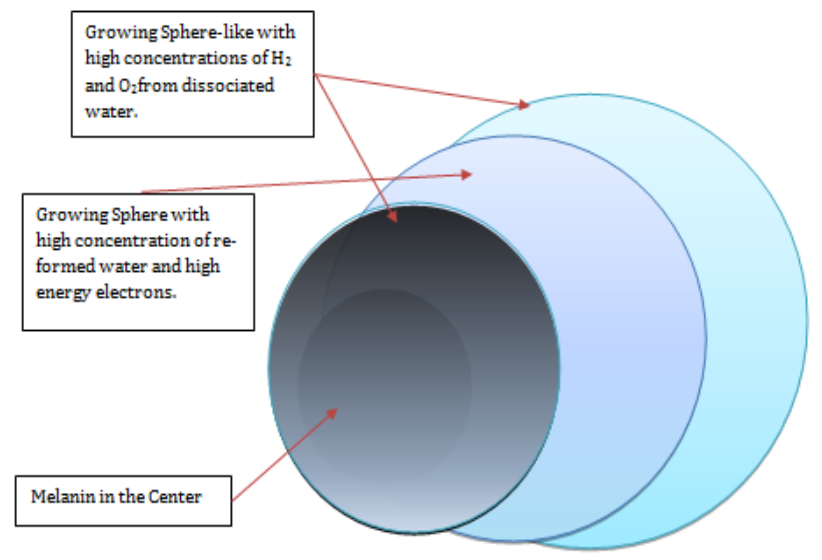

Figure I Melanin releases energy in symmetric form, in all directions; by way of growing energy spheres that differ between them in regards concentrations of hydrogen and oxygen and water re-formed with high energy electrons. In fact spheres grow themselves; we have them separated for purposes of clarity.

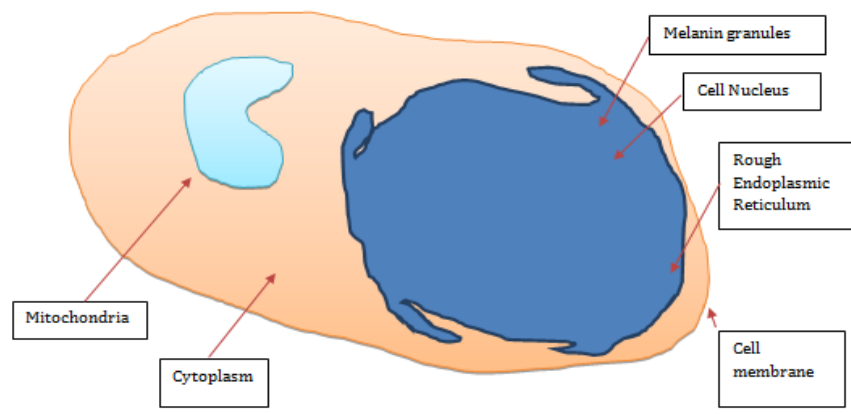

Figure 2 Two dimension scheme of the cell, showing the strategic position of the granules of melanin mainly inside the perinuclear space, which form and envelope around the cell nucleus, constituting its source of energy, recall the cell nucleus does not have mitochondria nor ATP, as well as the rough endoplasmic reticulum(the cell factory); that which completely surrounds the nucleus and melanosomes, allowing to the RER to capture the growing spheres of energy that emanating constantly, night and day from the melanin. Due to molecular hydrogen does not combined with water, this molecule moves easily through the cell cytoplasm following the laws of simple diffusion, thereby passing through the cell cytoplasm, flooding it completely and constantly, thereby, during $\mathrm{H}_{2}$ displacement; the different organelles may pick up the molecules of hydrogen, taking advantage of its energy load and its powerful antioxidant effect.

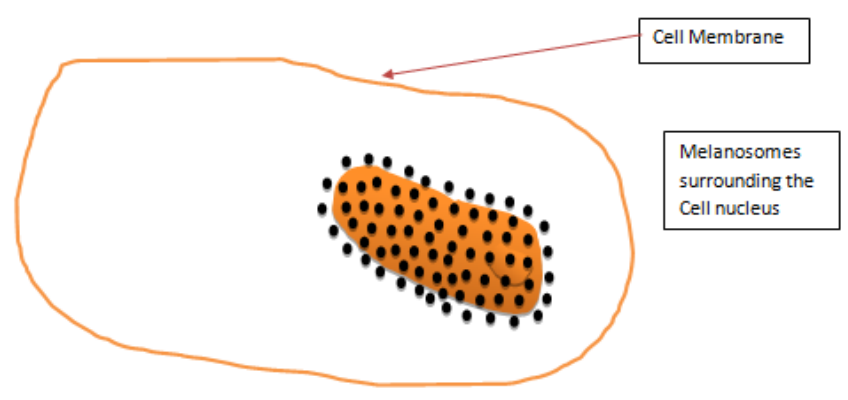

Figure 3 Three-dimensional representation of the aspect which would have the melanosomes surrounding the cell nucleus.

This unsuspected bioenergetics pathway allow us to discern the true function of the glucose as source of carbon chains, biomass, but not energy, as our body takes the energy of the light, visible and invisible; by means of the water molecule dissociation, like plants. Melanin granules, are strategically placed mainly in the perinuclear space (perikaryon), and completely surround the cell nucleus; what explains the operation of this organelle in spite of lacking mitochondria and ATP.

Perinuclear space and their melanosomes are, in turn, surrounded by the rough endoplasmic reticulum (RER); allowing this organelle to capture molecules of hydrogen that they emanate from melanin continuously, forming thus a source of energy, as RER has neither mitochondria nor ATP inside.

Melanin, wherever that it is, has the same biological role, energy production. And in the case of the turtle Trachemys and Chrysemys, the presence of melanin in the shell (Figure 4) explains its remarkable resistance to the low levels of oxygen, as melanin reveals that living beings are formed by cells energy independent, due its melanin content. The energy that emanates from the melanin in turtles, which mainly comes from the shell and skin, but not exclusively, because in fact all cells have melanin in varying degrees; is enough to maintain the system under anoxic conditions.

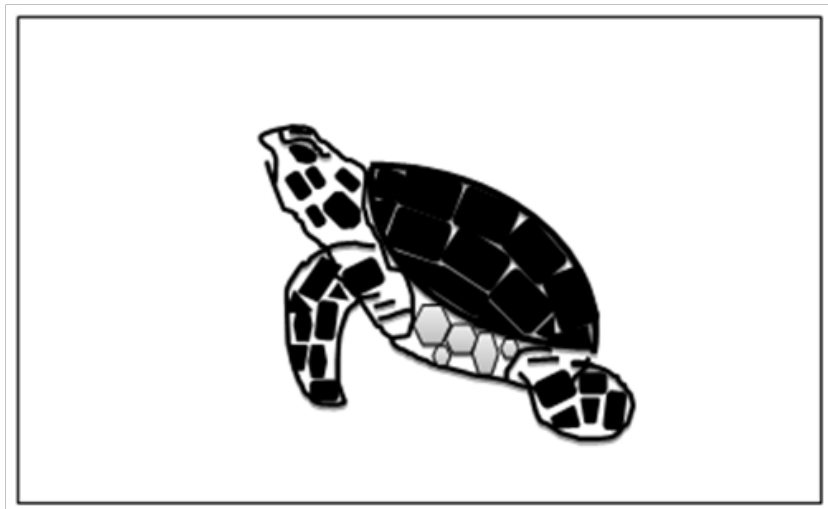

Figure 4 Turtles are reptiles characterized by bony or cartilaginous shell developed from their ribs and acting as a shield. This dome-shaped shell makes it difficult for predators to crush the shell between their jaws. In reality the shell acts as antenna which captures electromagnetic radiation.

Unsuspected intrinsic ability of melanin to dissociate the water molecule, leads to the need to rethink the physiology and biochemistry in very different bases, because then every cell in our body is able to produce its own chemical energy, which derives of light energy, and is converted into chemical energy by melanin through the dissociation of the water molecule.

This leads us to the following: living organisms need not carry oxygen from outside to inside through the hemoglobin, as each cell can produce it by herself. In the case of the tortoise, no need to take oxygen from the environment, from the atmosphere; melanin that is present in her body gives the ability to produce it through the dissociation of the water molecule.

So far had been considered that the shell of the turtle only functioned as a protection, as a defense against the bites of predators, although Jackson ${ }^{6}$ suggested that it also functioned as a $\mathrm{pH}$ buffer, because during prolonged anoxia, the shell and skeleton seems to releases carbonate buffers and captures lactic acid. ${ }^{6}$ For us, the shell is a way to optimize the capture of energy of electromagnetic radiation; other functions attributed to him are in addition. It is no coincidence 
that melanin is always at the top of the shell, what happens is that melanin tends to approach energy sources, in this case sunshine.

It is not by chance that melanin is located primarily in the upper part of living beings, is actually due to the shape and distribution of cells and the full body are determined by the generation and distribution of energy, as the whole universe.

Ionic Homeostasis, Melanin and neuron cell: The observed fact that both dopamine and glutamate continue to be released and taken back up into the cell during long term anoxia such that the turtle maintains basal extracellular levels, ${ }^{7}$ are processes that undoubtedly requires chemical energy available.

And this energy cannot come from ATP, since it is a parameter which decreases with anoxia; ${ }^{8}$ so the chemical energy required for that system not be disorganize and can keep in the minimum necessary so that recover quickly as soon as the conditions are right, has to come from somewhere; and the chemical energy that emanates from the melanin is a very suitable candidate.

The reuptake of neurotransmitters is energetically expensive, thereby, maintenance of its critical role implies an important energy cost. Much research on potential therapeutic interventions in stroke in humans has focused on blocking the release or ultimate effects of glutamate, including the tightly balance and reuptake of neurotransmitters.

In mammals, a moderate hypoxia (14\%) significantly increases the extracellular dopamine, even prior to depolarization..$^{9}$ On the contrary, in the turtle, both dopamine and glutamate maintain extracellular basal levels during anoxia which lasts for months.

Involves many variables in the process, why is that a response that allows to implement a treatment applicable to the human based in studies on the turtle could not be found. In the review of the literature, we find many studies, many different theories. It seems that wherever you look for, you will find something different, but ultimately does not answer the question, nor is not achieved to implement successful treatments in humans. So many alterations that happen in humans and that they do not occur in the turtles, would speak of widespread failure, which is characteristic of energy.

Thereby, promising in laboratory studies, such therapeutic interventions have been complete failures in clinical trials, ${ }^{10}$ suggesting a critical role for at least a minimum of glutamate cycling to maintain normal brain function. But this requires certain levels of available chemical energy, which may not come from ATP since also its production is diminished, both mammals and turtles.

The answer could be in the shell of the tortoise and its high content of melanin. Because the shell would run as a wide receiver of electromagnetic radiation. Consistently, the man, and even nonshelled reptiles do not show prodigious tolerance to anoxia of the turtles.

\section{Conclusion}

Melanin is able to transform light energy into chemical energy, and this has been accepted by the countries of the first world patent offices, ${ }^{11}$ which leaves no reasonable doubt about our finding. Still it is to understand how it uses eukaryote cell diatomic hydrogen and high energy electrons that are generated thanks to the dissociation of the water.

It is not an easy task since we are talking about millions of years of evolution, but now we have a firm base where starting, which would benefit patients affected by cerebral vascular events and other diseases.

In principle we must forget the sacred role of oxygen, and the facts show it: the turtle recovery requires more than just oxygen. Moreover, a turtle that hibernates under water has to leave the hypo metabolism before the restoration of the pulmonary oxygen, to be able to come to the surface and breathe. In our opinion, and given that melanin allows cells to produce its own oxygen through the dissociation of the water molecule, we think, in this moment; that the main function of breathing is to exhale $\mathrm{CO}_{2}$, more than transport the oxygen from the atmosphere to the interior of the body.

Upon the restoration of oxygen, blood $\mathrm{PO}_{2}$ recovers to normoxic levels within 10 mins. $^{12}$ But this oxygen comes from the water molecule dissociation, more that of the atmosphere.

It is conceivable that the processes that were initially downregulated and maintained at minimum levels should be intensified in a very coordinated way, it happens randomly. The process of ischemia/ anoxia, which is followed by re-oxygenation/reperfusion in mammals is known that it results in a transient and rapid increase of reactive oxygen species (ROS). ${ }^{13}$

But it is one of the properties of the molecular hydrogen, as it is the best antioxidant that is known. ${ }^{14}$ After hours or weeks of anoxia, the brain of the turtle should experience a massive insult of ROS during the re-oxygenation, but does not happen so. Conversely, the brain of mammals is very vulnerable to oxidative damage, probably due to their high levels of unsaturated fatty acids and iron, as well as relatively poor antioxidant defenses and that have no shell.

You may think that a greater amount of melanin in the organism means higher ability to resist hypoxia or even anoxia. Animals that hibernate have greater amount of melanin than the species that do not hibernate. But the amount of melanin does not determine the organism to overwinter or not, since this is determined by the circumstances of the environment and food availability. The organism uses the ability to hibernate when the circumstances so require.

The extraordinary intrinsic capacity of melanin transform light energy into chemical energy is perhaps the fundamental basis which allows the hibernation, as tissues gives the ability to resist the anoxia. So it is not strange that since 1914 reported that liver melanin content is not stable during the year in amphibians. They have shown significant morphological and functional changes in the hepatocytes of some amphibian species during the annual cycle; particularly in relation to the conditions of natural hibernation. ${ }^{15}$

Energy production being the main role of melanin in living organisms, then it is congruent to even increase during the winter, which has been reported on anuran and urodele species, ${ }^{16}$ within a range between 40 to $200 \%$ or even more.

The capacity of melanin transform light energy into chemical energy is attenuated with the cold and the low light, so the concept that hibernation is a physiological state in which cellular metabolism stays at its lowest seems correct, given that the main source of chemical energy, melanin decrease its energy production within certain ranges, which implies that biological systems will temper, but not destroyed.

Hibernation could be described as that systems and tissues of the body seem to be reduced to a minimum (Figure 5). They do not disappear, but they show significant changes, for example in the eye of the frog, the smooth endoplasmic reticulum of the pigmented epithelium of the retina, that is lush in summer, becomes scarce in winter, (Figure 6). ${ }^{17}$ 


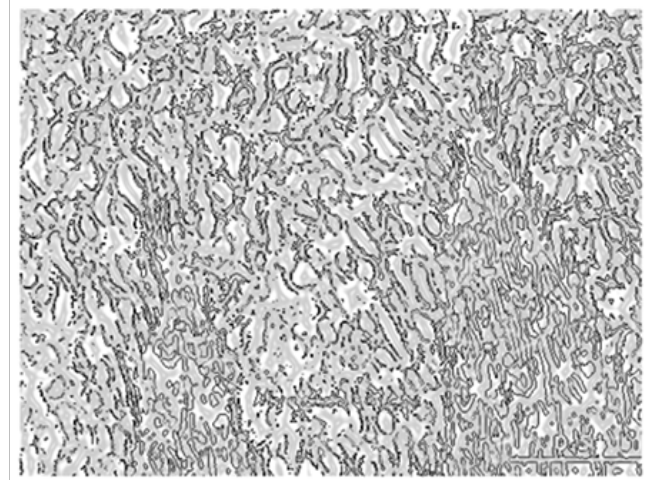

Figure 5 Smooth endoplasmic reticulum of cells of the retinal pigment epithelium of a frog in summer.

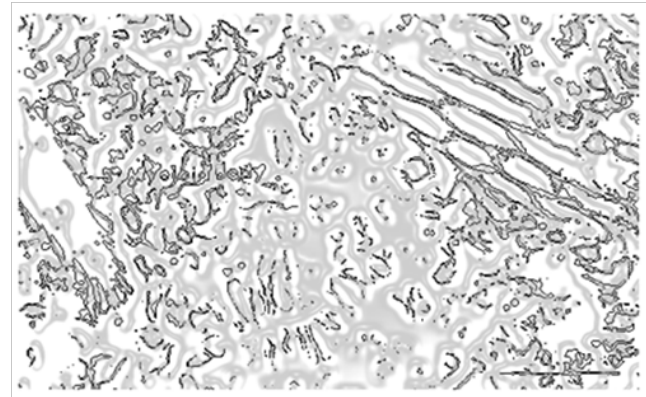

Figure 6 Smooth endoplasmic reticulum of cells of the Retinal pigment epithelium of a frog in winter.

Besides melanin, the cytoplasm of the retinal pigment epithelium (RPE) contains mainly smooth endoplasmic reticulum (SER), a highly metabolic organelle, as other important constituents such as mitochondria, rough endoplasmic reticulum, the nucleus, lysosomes, etc. Rod and cones, the visual elements of retina, are heavily dependent upon the RPE for nutritional support. And it is not by chance that cones and rods are immersed in a cell line that contains one of the concentrations of melanin higher body, the pigmented retinal epithelium. Both cones and rods as well as the RPE morphological characteristics are modified significantly during hibernation, because they seem to be reduced to a minimum, but maintain the characteristics and qualities that enable them to recover as soon as cold decreases and increases the amount of light.

Studies on the endocrinologic organs, pancreas and thyroid, also have shown remarkable morphologic changes ${ }^{18}$ Changes in the heart ${ }^{19}$ and kidney ${ }^{20}$ have also been reported.

In some organs, changes are very similar, but in others, they seem to be totally opposed, for example, the basement membrane (of Bruch) the RPE has not observable changes to electron microscopy, but the basal membrane of the renal glomerulus is thickened and increase their content of mucopolysaccharides. The above tells us that the changes that we can observe in the different tissues and cells depend on the location and function. The body takes them out very carefully in order to allow that it can maintain vitality with a minimum of metabolism and trying to preserve as much as possible the proper integrity. The ultimate goal is the body to awaken from hibernation when light and temperature conditions are suitable and recover its characteristics and qualities in the fastest way possible.

Hibernation organic changes can be described as if organisms are carefully dim. But to encompass all the cells, organs and systems, it is obvious that it is a highly complex process, very coordinated. Therefore it is difficult that it comes from a molecule in particular; called DNA, RNA, ATP, ADP, etc. But in the case of melanin is different, because the chemical energy that produces in the form of molecular hydrogen and high-energy electrons constitutes the common vital energy, that is completely usable for all and each of the organelles and processes which includes the eukaryotic cell, so, by reducing, in the case of hibernation, by cold and decrease in the amount of light it has a widespread effect but ordered at the same time on the metabolism of the cell. During hibernation, bodies approach at a necessary minimum level both in functional terms, such as biochemical and anatomical that allow them to survive until the conditions improve.

And something similar happens in the seeds (Figure 7), which are in a similar state of minimal vitality, but that hatch as the amount of water and light are appropriate. And in this we do not intervene genes or enzymes, because just when the generation of chemical energy that emanates from the melanin is sufficient, then all the reactions that form seed turned on and began to work for the chemical energy available is sufficient to fit all energy activation that is required by each one of the chemical reactions that make up what we call life. ${ }^{21}$ Melanin is ubiquitous in nature, is in all realms, including in fungi. ${ }^{22}$ And the concept that the main function of melanin is to transform light energy into chemical energy, ${ }^{23}$ begins to be accepted in the literature. ${ }^{24}$

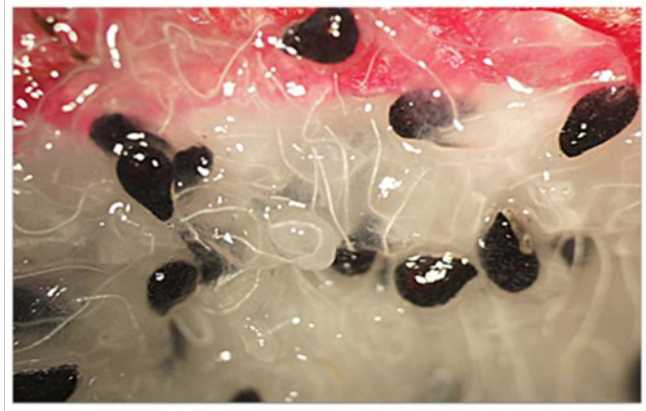

Figure 7 Pitaya seeds. The insistence of nature in place the melanin in all seeds, now is understandable.

Unveil the mystery of the hypoxia or anoxia tolerance, and understanding of the mechanisms of the animals that Hibernate, shall, without any doubt, a breakthrough in knowledge, which will result in better treatments for patients with chronic conditions likely to improve with strategies similar to those that uses nature since for thousands or millions of years.

\section{Acknowledgements}

None.

\section{Conflict of interest}

The author declares no conflict of interest.

\section{References}

1. Ultsch GR, Carwile ME, Crocker CE, et al. The physiology of hibernation among painted turtles: the eastern painted turtle, Chrysemys picta picta. Physiol Biochem Zool. 1999;72(4):493-501.

2. Jackson DC. Living without oxygen: lessons from the freshwater turtle. Comp Biochem Physiol A Mol Integr Physiol. 2000;125(3):299-315.

3. Lutz PL, Prentice HM. Sensing and responding to hypoxia, molecular and physiological mechanisms. Integ Comp Biol. 2002;42(3):463-468. 
4. Hong Y, Chen S, Zhang JM. Hydrogen as a selective antioxidant: a review of clinical and experimental studies. J Int Med Res. 2010;38(6):18931903.

5. Solis-Herrera A, Arias-Esparza MC, Solis-Arias RI, et al. The unexpected capacity of melanin to dissociate the water molecule fills the gap between the life before and after ATP. Biomed Res. 2010;21(2):224 226.

6. Jackson DC. Hibernating without oxygen: Physiological adaptations of the painted turtle. $J$ Physiol. 2002;543(Pt 3):731-737.

7. Milton SL, Lutz PL. Low extracellular dopamine levels are maintained in the anoxic turtle (Trachemys scripta) striatum. J Cereb Blood Flow Metab. 1998;18(7):803-807.

8. Milton SL, Lutz PL. Adenosine and ATP sensitive potassium channels modulate dopamine release in the anoxic turtle (Trachemys scripta) striatum. Am J Physiol Regul Integr Comp Physiol. 2005;289(1):R77R83.

9. Huang PL. Nitric oxide and cerebral ischemic preconditioning. Cell Calcium. 2004;36(3-4):323-329.

10. Hoyte L, Barber PA, Buchan AM, et al. The rise and fall of NMDA antagonists for ischemic stroke. Curr Mol Med. 2004;4(2):131-136.

11. Solis Herrera Arturo. Photoelectrochemical method of separating water into Hydrogen and Oxygen, using melanins or the analogues, precursors or derivatives thereof as the central electrolysing element. 2013. US 8455145 B2.

12. Fernandez A, Radmilovich M, Trujillo-Cenoz O. Neurogenesis and gliogenesis in the spinal cord of turtles. J Comp Neurol. 2002;453(2):131144.

13. Schild L, Reiser G. Oxidative stress is involved in the permeabilization of the inner membrane of brain mitochondria exposed to hypoxia/ reoxygenation and low micromolar $\mathrm{Ca}^{2+}$. FEBS J. 2005;272(14):35933601 .

14. Hong y, Chen S, Zhang JM. Hydrogen as a selective Antioxidant: a Review of Clinical and Experimental Studies. J Int Med Res. 2010;38(6):1893-1903.
15. Barni S, Paroni G, Vaccarone R, et al. Mechanisms of the liver pigmentation increase during the pre-hibernation of amphibians. European Journal of Histochemistry. 1997;41:19.

16. Barni S, Bertone V, Croce AC, et al. Increase in Liver pigmentation during natural hibernation in some amphibians. J Anat. 1999;195(1):1925 .

17. Kuwabara T. Cytologic changes of the retina and Pigment Epithelium during hibernation. Invest Ophtalmol. 1975;14(6):457-467.

18. Geuze JJ. Light and electron microscope observations on auto- and heterophagy in the exocrine pancreas of the hibernating frog (Rana esculenta). J Ultrastruct Res. 1970;32(5):391-404.

19. Zimny ML, Moreland JE. Mitochondrial populations and succinic dehydrogenase in the heart of a hibernator. Cana J Physiol Pharmacol. 1968;46(6):911-913.

20. Zimmy ML, Levy ED Jr. Ultrastructure of mesangial and juxtaglomerular cells in the kidney of a hibernator. Z Zellforsch Mikrosk Anat. 1971;118(3):326-332.

21. Solis Herrera A, Arias Esparza MC, Solís Arias MP. Photosynthesis in Humans. Chapter 12 of the book Photosynthesis. In: Nafees Khan, editor. Nova Science Publishers; 2015.

22. Dadachova E, Bryan RA, Howell RC, et al. The radioprotective properties of fungal melanin are a function of its chemical composition, stable radical presence and spatial arrangement. Pigment Cell Melanoma Res. 2008;21(2):192-199.

23. Solis-Herrera A, Arias-Esparza MC, Solís Arias, et al. Energy Production, the Main Role of Melanin in the Mesencephalon. J App Med Scs. 2013;2(2):11-20.

24. Brenner. Parkinson's Disease may be due to Failure of melanin in the substantia Nigra to produce molecular Hydrogen from dissociation of water, to protect the Brain from oxidative stress. Med Hypotheses. 2014;82(4):503. 